\title{
Will reconsolidation blockade offer a novel treatment for posttraumatic stress disorder?
}

\author{
Roger K. Pitman ${ }^{1,2 *}$ \\ Department of Psychiatry, Massachusetts General Hospital, Charlestown, MA, USA \\ 2 Department of Psychiatry, Harvard Medical School, Boston, MA, USA \\ *Correspondence: roger_pitman@hms.harvard.edu
}

Manyarticles about memory reconsolidation conclude with its therapeutic implications for posttraumatic stress disorder (PTSD). A core feature of PTSD is the memory of a traumatic event that is characterized by excessive strength, immalleability, and persistence. We found that Korean and World War II veterans with PTSD showed elevated physiological responses during mental imagery of their personal combat events as long as 40 years later (Orr et al., 1993). We have hypothesized that traumatic memories in PTSD become "overconsolidated" under the influence of stress hormones stimulated by the traumatic event (Pitman, 1989). Traditional theory holds that once a memory has been consolidated, i.e., placed into long-term storage, it exists as a permanent trace. According to this view, the most one can hope for therapeutically would be to inhibit the memory's expression through a mechanism such as extinction, but this inhibition is fragile, and the associated distress and arousal may return. Years ago we consulted on the case of a veteran who was admitted to the hospital for low back pain. Following World War II, he had experienced a year of nightmares and flashbacks of his combat experiences. With time these symptoms remitted, and he had been symptom free for 30 years. The medical work-up for his back pain revealed carcinoma of the prostate metastatic to the vertebrae, a fatal condition. The night after the patient was presented with this diagnosis, he experienced nightmares, not of his cancer or its future consequences, but of combat. This reinstatement of his combat memories by the stress of his cancer diagnosis indicated that they had not been erased but only had become latent.

Recent animal research has challenged the permanence of consolidated memory traces by suggesting that reactivation (retrieval) of a memory can return it to an unstable state from which it must be "reconsolidated" if it is to persist. Blocking reconsolidation offers the therapeutic possibility of weakening traumatic memories in PTSD. A recent Pavlovian differential conditioning study in normal humans employed memory reactivation accompanied by the beta-adrenergic blocker propranolol (Kindt et al., 2009). After that intervention, the previously acquired conditioned stimulus (CS) could no longer be made to elicit a skin conductance response. In contrast, the declarative memory of the contingency survived, suggesting that only the memory's fear component had been erased - an ideal scenario from the clinical standpoint. Another recent normal human conditioning study substituted a behavioral intervention (Schiller et al., 2010) and used potentiated startle as the measure of fear. A single CS trial was followed by a 10-min delay, and then by further extinction trials. Following this intervention, the conditioned fear response was not merely inhibited but permanently eliminated. It was argued that the delay provided sufficient time for the reactivated fear memory trace to return to an unstable state, so that the remaining CS presentations occurred during a "reconsolidation window." This allowed the original fear memory to be modified or "updated" to incorporate the new information that the CS was no longer dangerous. The investigators suggested that such a delay tactic could be incorporated into cognitive-behavioral therapy (CBT) to increase its efficacy. However, such a delay may already be a component of CBT, given that sessions typically go on for an hour or longer following the initial exposure (i.e., memory reactivation). Foa and Kozak (1986) have characterized the mechanism behind exposure therapy as the incorporation of "corrective information."

Although the preclinical animal and normal human studies are encouraging, the translational gap to clinical application is huge. Critical differences between PTSD and laboratory experiments include (in the former) the stronger unconditioned stimulus (US, e.g., a gunshot wound vs. a mild electric shock), greater and more sustained arousal at the time of the traumatic event (i.e., a stronger unconditioned emotional response), the more complex nature of the CS (e.g., a firefight vs. a colored shape), the possible presence of multiple conditioning events, and the longer duration between the memory's formation and the intervention (e.g., years vs. days). Erasing or updating the memory of a conditioned response acquired 1 day earlier under the influence of a mild US might be likened to the effect of a firecracker, whereas achieving the same for a deeply engraved traumatic memory of a life-threatening event in PTSD might be likened to the effect of an atomic bomb. It remains to be seen whether such a device can be constructed.

Lang (1985) proposed that emotion is defined by a specific information structure in memory, whose content consists of three primary categories: (1) information about prompting external stimuli and the context in which they occur (stimulus propositions); (2) information about responding in this context, including expressive verbal behavior, overt acts, and the visceral and somatic events that mediate arousal and action (response propositions); and (3) information that defines the meaning of the stimulus and response data (meaning propositions). These propositions are organized into an associative network which, when a critical number of propositions are accessed, is processed as a unit. We suggested that PTSD consists of one or more traumatic emotion networks that, when activated, produce its characteristic symptomatology (Pitman, 1988; Pitman and Orr, 1990). If a PTSD associative network could be reactivated in its entirety, and then have its reconsolidation blocked in entirety, this could simplify clinical application. Unfortunately research with second-order conditioning suggests that this may not be 
so simple. Dębiec et al. (2006) conditioned rats to a tone pattern (CS1) by pairing it with a shock US (first-order conditioning). Then they conditioned rats to different tone pattern (CS2) by pairing it with the CS1 (second-order conditioning). They found that blocking reconsolidation of the firstorder association with the protein-synthesis inhibitor anisomycin reduced the freezing (fear) response to both the CS1 and the CS2. In contrast, blocking reconsolidation of the second-order association reduced the freezing response only to the CS2; the freezing response to the CS1 remained intact. These findings suggest that successful reconsolidation blockade or memory updating in PTSD will require accessing the original, core traumatic associations; merely addressing secondary, peripheral associations will not suffice. Moreover, under certain circumstances (Eisenberg et al., 2003), pharmacological intervention could succeed not in blocking reconsolidation of the fear association but rather in blocking consolidation of extinction learning, possible resulting in an antitherapeutic effect.

Unfortunately from the therapeutic application standpoint, animal evidence indicates memories that have been formed under stressful conditions (Bustos et al., 2010), as well as memories that have aged for long periods (Suzuki et al., 2004), are more resistant to being made to undergo reconsolidation. For reconsolidation blockade, or updating, to be successful, two steps are required. First, the problematic memory must be destabilized. Second, its restabilization (reconsolidation) must then be prevented or modified (updated). Resistance may be encountered during the first of these stages. Specifically, activation of NR2B NMDA-receptor subunits appears to be required for reactivation-induced memory destabilization, and their downregulation may prevent this. Recent animal research suggests that administration of the NMDA agonist D-cycloserine (DCS) may prepare a memory for destabilization and facilitate pharmacological reconsolidation blockade that would otherwise not take place (Bustos et al., 2010). Given that both the formation of memories under stressful conditions, and the age of such memories, characterize PTSD, for a reconsolidation-based treatment to work, pharmacological or other assistance with memory destabilization may be required, in addition to subsequent pharmacological blockade of reconsolidation, or behavioral updating, once destabilization has occurred. This suggests a second possible, and different, application of DCS to PTSD therapy, in addition to its possible role in the strengthening of extinction retention (Cukor et al., 2009).

The only published reconsolidation blockade-like study in PTSD to date did succeed in producing evidence that propranolol administered at the time of traumatic memory reactivation diminished the memory's emotional component, as manifest in smaller psychophysiological responses during subsequent script-driven traumatic imagery (Brunet et al., 2008). However, this study lacked sufficient controls to permit the inference that reconsolidation blockade was the underlying mechanism. Moreover, these results are preliminary, and many additional studies will be required to determine whether the therapeutic promise of reconsolidation blockade or modification will be fulfilled.

Finally, there is no reason to assume that if the therapeutic PTSD bomb can eventually be constructed, its ultimate ingredient will be propranolol. In unpublished research with rats, we have found that the glucocorticoid receptor antagonist mifepristone, in addition to blocking reconsolidation of inhibitory avoidance learning (Taubenfeld et al., 2009), has substantially stronger cue-induced-fear reconsolidation-blocking properties than propranolol. This drug has yet to be tested in human reconsolidation experiments. Other drugs may exist that are stronger still. However, for any drug to be clinically useful, it must be approved for human use and capable of systemic administration. Moreover, the efficacy of any drug has yet to be compared with the efficacy of behavioral memory updating techniques. All these questions and more will need to be addressed within a large research and development Manhattan project for PTSD. The translation from preclinical work to clinical application may prove long and difficult, and even unsuccessful. However, given the importance of PTSD as a public mental health problem, it is worth pursuing.

\section{REFERENCES}

Brunet, A., Orr, S. P., Tremblay, J., Robertson, K., Nader, K., and Pitman, R. K. (2008). Effect of post-retrieval propranolol on psychophysiologic responding during subsequent script-driven traumatic imagery in post-traumatic stress disorder. J. Psychiatr. Res. 42, 503-506.

Bustos, S. G., Giachero, M., Maldonado, H., and Molina, V. A. (2010). Previous stress attenuates the susceptibility to midazolam's disruptive effect on fear memory reconsolidation: influence of pre-reactivation D-cycloserine administration. Neuropsychopharmacology 35, 1097-1108.

Cukor, J., Spitalnick, J., Difede, J., Rizzo, A., and Rothbaum, B. O. (2009). Emerging treatments for PTSD. Clin. Psychol. Rev. 29, 715-726.

Dębiec, J., Doyère, V., Nader, K., and Ledoux, J. E. (2006). Directly reactivated, but not indirectly reactivated, memories undergo reconsolidation in the amygdala. Proc. Natl. Acad. Sci. U.S.A. 103, 3428-3433.

Eisenberg, M., Kobilo, T., Berman, D. E., and Dudai, Y. D. (2003). Stability of retrieved memory: inverse correlation with trace dominance. Science 301, 1102-1104.

Foa, E. B., and Kozak, M. J. (1986). Emotional processing of fear: exposure to corrective information. Psychol. Bull. 99, 20-35.

Kindt, M., Soeter, M., and Vervliet, B. (2009). Beyond extinction: erasing human fear responses and preventing the return of fear. Nat. Neurosci. 12, 256-258.

Lang, P. J. (1985). “The cognitive psychophysiology of emotion: fear and anxiety," in Anxiety and the Anxiety Disorders, eds A. H. Tuma and J. Maser (Hillsdale, NJ: Lawrence Erlbaum Associates), 131-170.

Orr, S. P., Pitman, R. K., Lasko, N. B., and Herz, L. R. (1993). Psychophysiological assessment of posttraumatic stress disorder imagery in World War II and Korean combat veterans. J. Abnorm. Psychol. 102, 152-159.

Pitman, R. K. (1988). Post-traumatic stress disorder, conditioning, and network theory. Psychiatr. Ann. 8, 182-189.

Pitman, R. K. (1989). Post-traumatic stress disorder, hormones, and memory. Biol. Psychiatry 26, 221-223.

Pitman, R. K., and Orr, S. P. (1990). The black hole of trauma. Biol. Psychiatry 27, 469-471.

Schiller, D., Monfils, M. H., Raio, C. M., Johnson, D. C., Ledoux, J. E., and Phelps, E. A. (2010). Preventing the return of fear in humans using reconsolidation update mechanisms. Nature 463, 49-53.

Suzuki, A., Josselyn, S. A., Frankland, P. W., Masushige, S., Silva, A. J., and Kida, S. (2004). Memory reconsolidation and extinction have distinct temporal and biochemical signatures. J. Neurosci. 24, 4787-4795.

Taubenfeld, S. M., Riceberg, J. S., New, A. S., and Alberini, C. M. (2009). Preclinical assessment for selectively disrupting a traumatic memory via postretrieval inhibition of glucocorticoid receptors. Biol. Psychiatry 65, 249-257.

Received: 19 September 2010; accepted: 23 February 2011; published online: 04 March 2011.

Citation: Pitman RK (2011) Will reconsolidation blockade offer a novel treatment for posttraumatic stress disorder? Front. Behav. Neurosci. 5:11. doi: 10.3389/ fnbeh.2011.00011

Copyright (c) 2011 Pitman. This is an open-access article subject to an exclusive license agreement between the authors and Frontiers Media SA, which permits unrestricted use, distribution, and reproduction in any medium, provided the original authors and source are credited. 